\section{Cytomegalovirus colitis in an HIV-positive woman with a relatively preserved CD4 count}

\section{To the Editor:}

Cytomegalovirus (CMV) disease typically occurs in immunocompromised individuals including HIV-positive patients with CD4 counts less than 50 cells $/ \mu \mathrm{L}$ to 100 cells $/ \mu \mathrm{L}$ (1). There have been few cases of CMV gastrointestinal disease reported in patients with CD 4 counts greater than 50 cells $/ \mu \mathrm{L}$ to 100 cells $/ \mu \mathrm{L}$ $(2,3)$. We describe a case of CMV colitis in an HIV-infected patient with a relatively preserved CD4 count.

A 39-year-old woman with known HIV infection, hepatitis C virus infection, alcoholism and injection drug use was admitted to hospital in August 2006 with a decreased level of consciousness secondary to an overdose of methadone. Acutely, her methadone overdose was treated with supportive care, and she received therapy for concurrent aspiration pneumonia.

Once alert, she complained of dysphagia, epigastric pain and nonlocalizing lower abdominal pain. She appeared wasted and described weight loss over the preceding year for which she had not sought medical care. She attributed her weight loss to poor oral intake and chronic watery diarrhea but denied fevers or night sweats. She had never maintained consistent follow-up for her HIV infection and other comorbidities due to a transient lifestyle. She had no known history of opportunistic infections and had never received antiretroviral therapy. Her CD4 count at the time of hospitalization was 340 cells/ $/ \mathrm{L}$ (34\%).

Investigations revealed endoscopically and microbiologically documented Candida esophagitis. Biopsies of the duodenum and small bowel revealed mild inflammation without evidence of mycobacterial disease or CMV on histopathology. Colonoscopy revealed diffuse inflammation and mucosal ulceration, but the histopathology was nondiagnostic. Specifically, there was no evidence of CMV colitis. Mycobacterial and fungal cultures of the colon were also negative. Stool samples were negative for Clostridium difficile toxin, pathogenic bacteria and parasites including Microsporidia, Isospora, Cryptosporidia and Cyclospora. There was no clinical, laboratory or radiographic evidence of cirrhosis, and sputum and blood cultures for Mycobacterium species were negative. She received oral fluconazole for esophageal candidiasis and her dysphagia improved, although her anorexia and abdominal discomfort persisted. She was discharged and outpatient follow-up with Gastroenterology and Infectious Diseases was arranged.

The patient missed her follow-up appointments and returned to hospital three weeks later with worsening abdominal pain and persistent anorexia. She had clinical evidence of peritonitis and radiological evidence of a perforated viscus. She was taken to the operating room where multiple small and large bowel perforations were found. She required a right hemicolectomy and underwent an abdominal wash-out. Pathology was consistent with CMV colitis with numerous CMV inclusion bodies identified throughout all biopsies of small and large bowel. Biopsy cultures were negative for mycobacteria and fungi. Repeat stool examinations were negative for Clostridium difficile toxin, pathogenic bacteria and parasites. A repeat CD4 count at this second admission was 270 cells/ $\mu \mathrm{L}$ (34\%), with an HIV viral load of 13,000 RNA copies/mL. Review of her CD4 counts over time confirmed that her CD4 count had never been below 270 cells/ $\mu \mathrm{L}$.
She was treated with intravenous ganciclovir $5 \mathrm{mg} / \mathrm{kg}$ twice daily for 20 days and improved. She was discharged with follow-up with a plan to begin highly active antiretroviral therapy as an outpatient.

Two cases of HIV-infected patients with CMV colitis and relatively preserved CD4 counts in the setting of malnutrition and alcoholism have been described previously $(2,3)$. Malnutrition has long been associated with anergy and impaired $\mathrm{T}$ cell function, thereby potentially predisposing patients to reactivation of CMV (4). Indeed, there are case reports of CMV disease in malnourished individuals, who were otherwise apparently immunocompetent $(5,6)$. The exact mechanism by which this would lead to enhanced CMV reactivation is unknown. Another commonality between our case and the two previous case reports was the presence of esophageal candidiasis. Yotsumoto et al (2) hypothesized that candidiasis may damage the mucosa leading to impairment of the local immunological defense system resulting in local CMV disease.

It is clinically important not to miss the diagnosis of CMV colitis as effective treatment is available. Typically, patients are treated with either a course of intravenous ganciclovir or foscarnet, for 21 to 28 days (7). Secondary prophylaxis is not routinely recommended for gastrointestinal disease but should be considered if relapses occur (7). It should be noted that an episode of CMV colitis is evidence of symptomatic HIV infection and is thus an indication for initiation of highly active antiretroviral therapy (7).

Although gastrointestinal manifestations of CMV in patients with advanced HIV disease are well described, our case highlights the fact that CMV colitis can also occur in patients with relatively preserved CD4 counts (ie, CD4 counts of 250 cells/ $\mu \mathrm{L}$ to 350 cells/ $/ \mathrm{L}$ ), and should be included in the differential diagnosis of any HIV-positive patient presenting with lower gastrointestinal symptoms, regardless of their CD4 counts. Significant alcohol use, malnutrition and Candida esophagitis may predispose to CMV colitis in HIV-positive patients.

\section{Jennie Johnstone $\mathrm{MD}$, Curtiss R Boyington MD, Lilly J Miedzinski MD, Isabelle Chiu MD University of Alberta, Edmonton, Alberta}

\section{REFERENCES}

1. Springer KL, Weinberg A. Cytomegalovirus infection in the era of HAART: fewer reactivations and more immunity. J Antimicrob Chemother 2004;54:582-6.

2. Yotsumoto M, Nakamura N, Kitano K, et al. Cytomegalovirus esophagitis and colitis, esophageal candidiasis and colon amebiasis

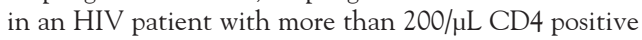
lymphocytes. J AIDS Res 2002;5:153-7.

3. Wolf T, Bickel M, Faust D, Fellbaum C, Brodt HR. A case of severe CMV colitis in an HIV positive patient despite moderate immunodeficiency. Scand J Infect Dis 2003;35:904-6.

4. Christou NV, Meakins JL, Gordon J, et al. The delayed hypersensitivity response and host resistance in surgical patients: 20 years later. Ann Surg 1995;222:534-46.

5. Boyce TG, Wright P. Cytomegalovirus pneumonia in two infants recently adopted from China. Clin Infect Dis 1999;28:1328-30.

6. Barrio VR, Darmstadt GL. Rash and opportunistic pneumonia in a malnourished infant adopted from China. Clin Infect Dis 2000;30:408-9.

7. Centers for Disease Control and Prevention. Treating opportunistic infections among HIV-infected adults and adolescents:

Recommendations from CDC, the National Institutes of Health, and the HIV Medicine Association/Infectious Diseases Society of America. MMWR 2004;53 (No. RR-15):1-112. 


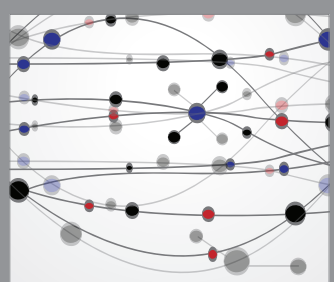

The Scientific World Journal
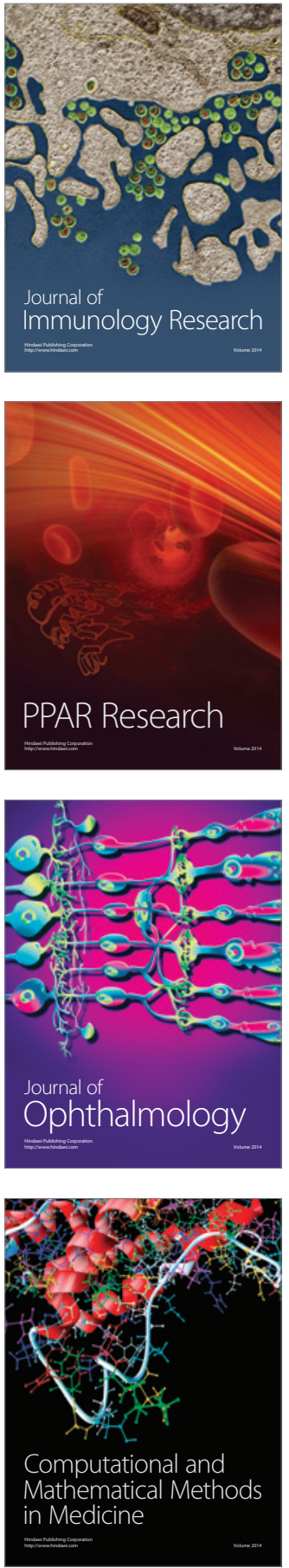

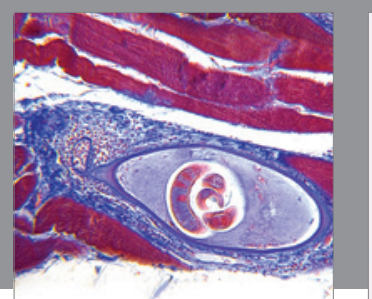

Gastroenterology Research and Practice

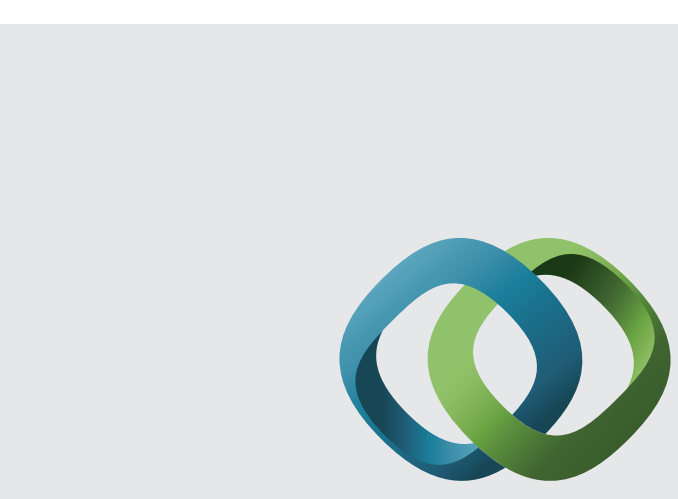

\section{Hindawi}

Submit your manuscripts at

http://www.hindawi.com
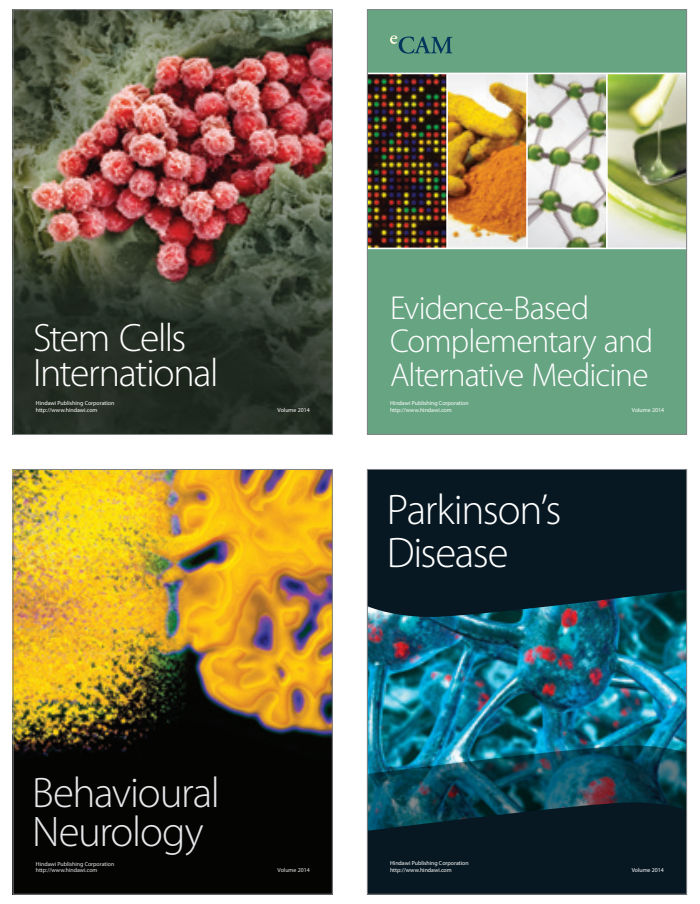
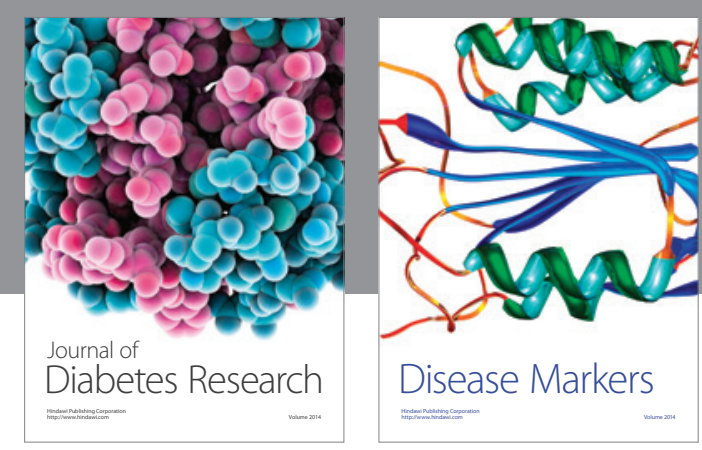

Disease Markers
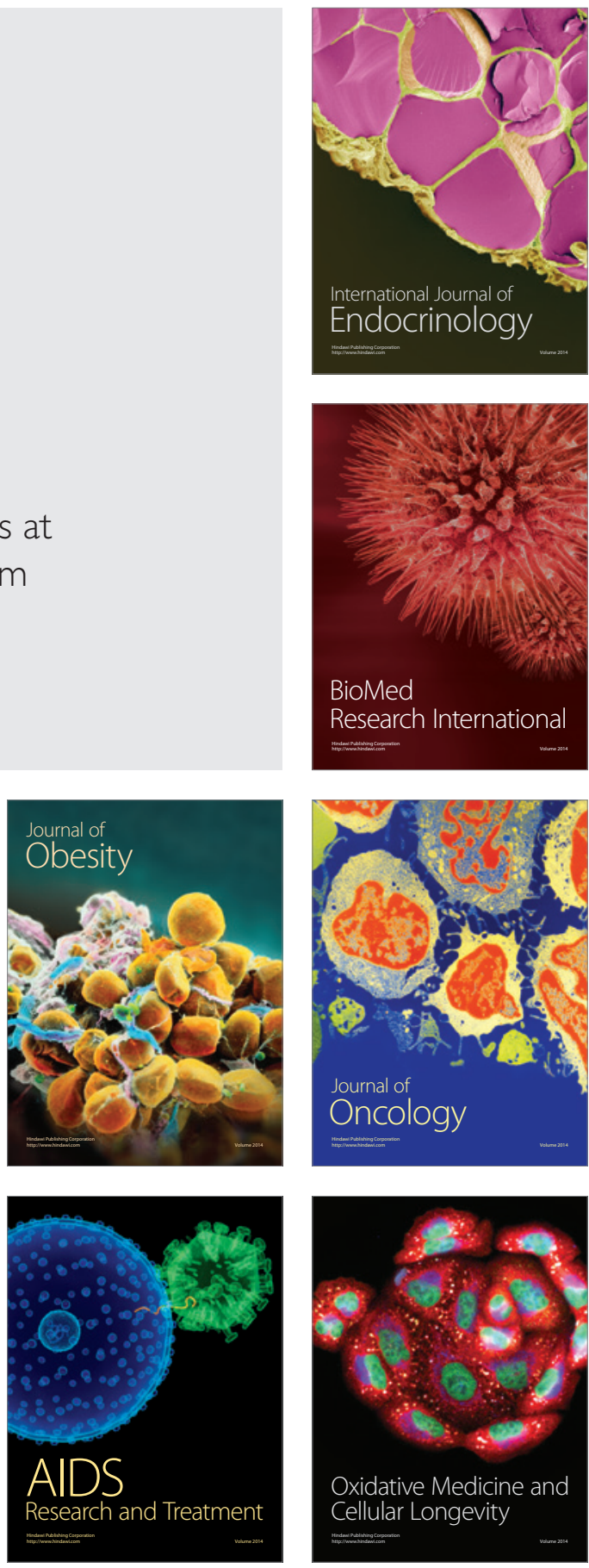